\title{
Frozen water waves over rough topographical bottoms
}

\author{
Liang-Shan Chen $^{1}$ and Zhen $\mathrm{Ye}^{1,2 *}$ \\ ${ }^{1}$ Department of Physics, Fudan University, Shanghai, \\ China and ${ }^{2}$ Wave Phenomena Laboratory, Department of Physics, \\ National Central University, Chungli, Taiwan, China
}

\begin{abstract}
The propagation of surface water waves over rough topographical bottoms is investigated by the multiple scattering theory. It is shown that the waves can be localized spatially through the process of multiple scattering and wave interference, a peculiar wave phenomenon which has been previously discussed for frozen light in optical systems (S. John, Nature 390, 661, (1997)). We demonstrate that when frozen, the transmission of the waves falls off exponentially, and a cooperative behavior appears, fully supporting previous predictions. A phase diagram method is used to illustrate this distinct phase states in the wave propagation.

PACS numbers: $47.10 .+\mathrm{g}, 47.11 .+\mathrm{j}, 47.35 .+\mathrm{i}$.
\end{abstract}

Multiple scattering occurs when waves propagate in media with scatterers, leading to many interesting phenomena such as the bandgaps in periodically structured media and Anderson localization in disordered media [1]. Within a bandgap, waves are evanescent; when localized, they remain trapped spatially until dissipated. The phenomenon of bandgaps and localization has been both extensively and intensively studied for electronic, electromagnetic, and acoustic systems 2].

Recently, Wiersma et al. [3] have demonstrated that light can be forced to stand still in strongly scattering semiconductor powders. The authors have shown a transmission transition from classical diffusion to localization. When localized, the optical transmission decays exponentially instead of linearly with the thickness of a sample. At the transition, the transmission has a power-law dependence on the inverse thickness. This experiment not only leads to new applications [4] in optical data processing and laser action, but also sheds new lights to the understanding of the Anderson localization transition. Although regretfully not being shown by this experiment, it has been further predicted by John [4] that a coherent behavior should appear for localized waves. In addition, in the classical diffusion through a scattering medium, the intensity distribution will fluctuate significantly due to wave interference, while the fluctuation will be reduced in the localized state.

Here we report that the Anderson localization phenomenon may also be observed for the surface water wave propagation over random bottoms, also termed as gravity waves in fluid mechanics. We show that gravity waves can come to a complete halt in the presence of random multiple scattering and wave interference. In the localized state, not only the wave intensity decreases exponentially in agreement with the observation in Ref. [3] , but also a distinct phase coherence behavior prevails. The

\footnotetext{
*Corresponding author: zhen@phy.ncu.edu.tw
}

transition from the diffusive to localization regime is signified by the variations in the fluctuation of the transmission. The localization regime overlaps partially with the bandgap of corresponding regularly structured bottoms. These observations fully support the predictions in Ref. [4], indicating that these phenomena are a generic property of waves.

The propagation of water waves over topographical bottoms has actually been a subject of much research, from both ocean engineering and fundamental research perspectives (e. g. Refs. [5, [6, 7, 8, 9] ). A comprehensive summary and reference can be found in excellent textbooks 10, 11, 12].

The concept of Anderson localization has also been extended to the study of the propagation of surface water waves over rough bottoms. In 1983, Guazzelli et al. 13. suggested that the phenomenon of Anderson localization could be observed on one dimensional shallow water waves, when the bottom has random structures. Later, Devillard et al. reconsidered the problem by the potential theory [14]. The experimental observation of water wave localization has been subsequently suggested by Belzone et al. 15. These earlier attempts have been limited to the transmission measurement and to one dimension.

A recent experiment 16] has used water waves to illustrate the Bloch wave phenomenon over a two dimensional periodic bottom. This pioneering experiment has made it possible that the abstract concept be presented in an unprecedentedly clear manner.

The experimental advantures [15, 16] pave a new avenue for investigating the phenomena of Anderson localization in disordered media and wave bandgaps in periodic structures. These developments are the motivation for us to explore some important properties of Anderson localization in the propagation of surface water waves over random topographical bottoms.

Making the results experimentally testable, we will adopt the systems from the experiment 16]. The conceptual layout of the systems is illustrated in Fig. 1 We consider a water column with a uniform depth $H$. There 
are $N$ cylindrical steps mounted on the water bottom. For simplicity, the steps are assumed to be identical. The heights of the steps are denoted by $\Delta H$ and the radii are $a$; we can also extend to consider the situation of cylindrical dimples by letting $\Delta H<0$. For comparison, we will consider both the randomly and the corresponding orderly arrangements of the steps on the bottoms. In the ordered case, the steps form a square lattice with the lattice constant $d$; therefore the areal occupation fraction by the steps is $\beta=\frac{\pi a^{2}}{d^{2}}$. In the random case, the steps are placed completely randomly within a circular area of radius $R$. In both cases, the areal occupation fraction is same; therefore $L=\sqrt{\left(N \pi a^{2}\right) / \beta}$ and $R=\sqrt{\left(N a^{2}\right) / \beta}$. A monochromatic transmitting source of angular frequency $\omega$ is located in the middle of the arrays of the steps. The transmission is measured by a receiver located outside the arrays. The water surface is in the $x-y$ plane. In the simulation, all lengths are scaled by the lattice constant $d$. This is a two dimensional problem.

( a )

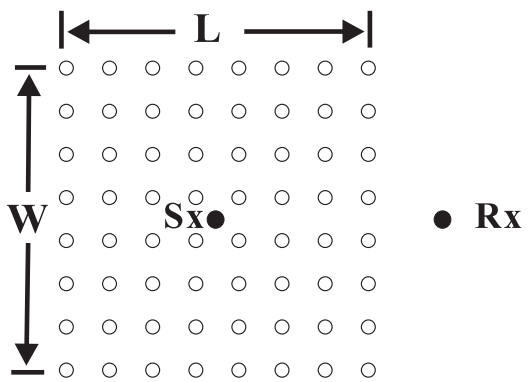

( b )

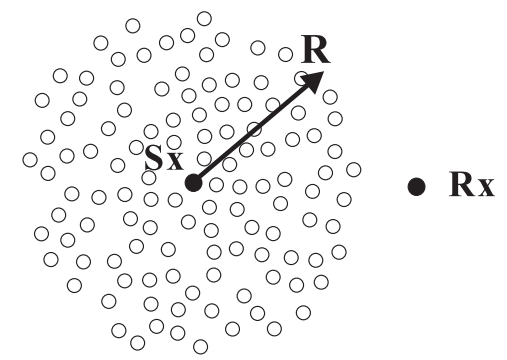

(c)

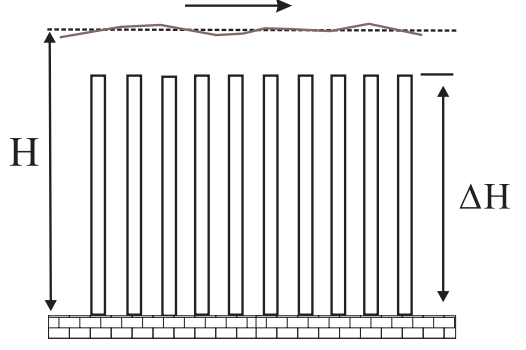

FIG. 1: The conceptual layout of the systems: (a) and (b) show the bird's views; and (c) the side view. The circles denote the cylindrical steps, while $\mathrm{Sx}$ and $\mathrm{Rx}$ refer to the stimulating source and the receiver respectively.

The governing equations for the motion of surface water waves in the systems described by Fig. 11 can be obtained by invoking the Newton's second law and the con- servation law of mass. The formulation has been given in Ref. 16, 17] and the detailed derivation is given in Ref. [18]. Here we only list the final equations.

The displacement of the water surface is denoted by $\eta(\vec{r}, t)$. The equation of motion for its Fourier component is derived as [16, 17, 18]

$$
\nabla\left(\frac{1}{k^{2}} \nabla \eta(\vec{r})\right)+\eta(\vec{r})=-4 \pi \delta\left(\vec{r}-\vec{r}_{s}\right),
$$

where $\nabla=\partial_{x} \vec{e}_{x}+\partial_{y} \vec{e}_{y}, \vec{r}_{s}$ is the location of the transmitting source, and the wavenumber $k$ satisfies

$$
\omega^{2}=\left(g k(\vec{r})+b k^{2}(\vec{k})\right) \tanh (k(\vec{r}) h(\vec{r})),
$$

in which $b$ is the capillary length. For a fixed frequency $\omega$, the wavenumber varies as a function of the depth $h(\vec{r})$. Without the steps, the wave field is $\eta_{0}$. We note that Eq. (II) is derived when non-linear effects are ignored. The non-linearity may give rise to delocalization [9].

In this paper we will apply Eq. (1) to the systems depicted in Fig. 11 The transmitted waves will be scattered repeatedly by the steps, forming an orchestral pattern of multiple scattering. Such a multiple scattering process can be solved for any arrangement of the steps by the multiple scattering theory [18] following the work of Twersky [19]. In the computation, the transmission is normalized such that it is unity when there are no scatterers, thus eliminates the trivial geometrical spreading effect In the periodic case, the plane wave expansion method will be used to compute the band structures of the water waves 18 .

A set of numerical simulations has been carried out. In the simulation, the following parameters are adopted from the experiment [16]: the depth $H=2.5 \mathrm{~mm}$; the height of the cylinders $\Delta H=2.49 \mathrm{~mm}$; in the periodic case, the lattice constant $d=2.5 \mathrm{~mm}$; the radius of the steps $a=0.75 \mathrm{~mm}$; the capillary length $b=0.93 \mathrm{~mm}$. In the random case, the transmission intensity is averaged over the random configurations.

First, in Fig. 2] we show the normalized intensity of transmitted waves $\left(|T|^{2}=\left|\eta / \eta_{0}\right|^{2}\right)$ as a function of frequency, and the band structure of the corresponding square arrays of the cylindrical steps. When computing the transmission, the receiver is located at two lattice constants away from the arrays. To ensure the stability of the results, enough modes and number of steps have been considered. For instance, the maximum mode number and the maximum array size considered are 9 and $14 \times 14$ respectively. The wave transmission along the $\Gamma X$ direction is shown for the periodic case. The transmission through the random arrays of steps is also plotted. In both cases, the source is placed in the middle of the arrays.

Here we see that a complete bandgap region can be identified as ranging from about 12.5 to $16 \mathrm{~Hz}$. A strong localization regime is shown to range from 10 to $14 \mathrm{~Hz}$ in 
the random case. Though shallower, this regime overlaps with the inhibition regime in the periodic case. By comparison, we see that although not exactly overlapping with each other, the strong localization and complete bandgap regime are close to each other; thus finding the complete bandgaps will facilitate locating the localization regimes, as suggested in Ref. [20]. We note here that in the periodic case the inhibition regime in the transmission does not fully overlap with the complete bandgap. This is because we have put the source inside the array. When the transmission is measured across the sample, i. e. the source is placed on one side of the sample and the receiver is placed on the other, the two will overlap.
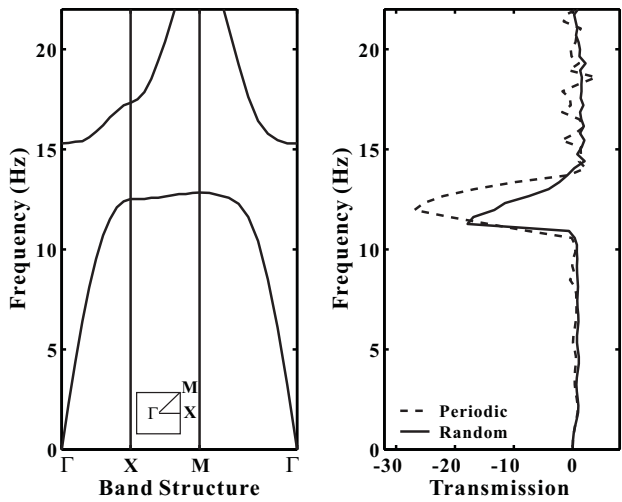

FIG. 2: Right Panel: Normalized transmission $\ln |T|^{2}$ versus frequency for the complete random arrays (solid line) and the corresponding square lattice of cylindrical steps (dotted line). The transmission in the periodic case is along the $\Gamma X$ direction. Left panel: the band structures for the square lattice. The inserted box in (a) denotes the Brillouin zone.

To show the conjectured frozen feature of localized waves and fluctuating feature of non-localized waves [4], we have considered two frequencies: one is in the passing band $5 \mathrm{~Hz}$, and the other is with the complete bandgap region, $11.966 \mathrm{~Hz}$. We write the wave field $\eta(\vec{r})$ as $|\eta(\vec{r})| e^{i \theta(\vec{r})}$. This allows us to separate the amplitude field and the phase field of the waves. For the phase field $\theta$, we define a unit phase vector field as $\vec{u}=\cos \theta \hat{e}_{x}+\sin \theta \hat{e}_{y}$. Both phase vector field and the wave intensity field $|\eta|^{2}$ can be plotted in the $x-y$ plane. The significance of the phase vector field is as follows. The intensity flux of surface water waves can be shown as $\vec{j} \sim|\eta|^{2} \nabla \theta$. It is clear that when $\theta$ is constant, at least by spatial domains, while $|\eta| \neq 0$, the wave transport would come to a stop and the waves will be localized or frozen in the space.

In Fig. 3 the two-dimensional spatial distribution of the normalized intensity $\left|\eta / \eta_{0}\right|^{2}$ and the phase vector field are plotted for the two frequencies. The phase vectors are located randomly in the $x-y$ plane. For $f=5$ $\mathrm{Hz}$, the intensity spreads spatially, meanwhile the phase vectors point to various directions. This indicates that waves are not yet localized at this frequency. The results in Fig. 3(b) nicely demonstrate the properties of the localized or frozen waves. At $f=11.966 \mathrm{~Hz}$, the wave intensity is mainly confined near the transmission site. Meanwhile, there is an ordering in the phase vector field, that is, all the phase vectors either point to the same direction or the opposite direction, indicating that the phase field is constant by domains. Here we clearly demonstrate that the localized waves behave as a standing wave in the random media. These observations fully comply with the above general discussion of localization, and also support the previous predictions. The intensity distribution in Fig. 3 realizes remarkably well what has been conjectured in Fig. 1 of Ref. [4]. We note that the disorientation at the boundary is due to the finite size in the simulation. For a finite system, the wave can leak out at the boundary, resulting in disappearance of the phase coherence. When enlarging the sample size, we observe that the area showing the perfect phase coherence will increase. We have further verified that the features shown in Fig. 3 remain quantitatively the same for any other random configuration.
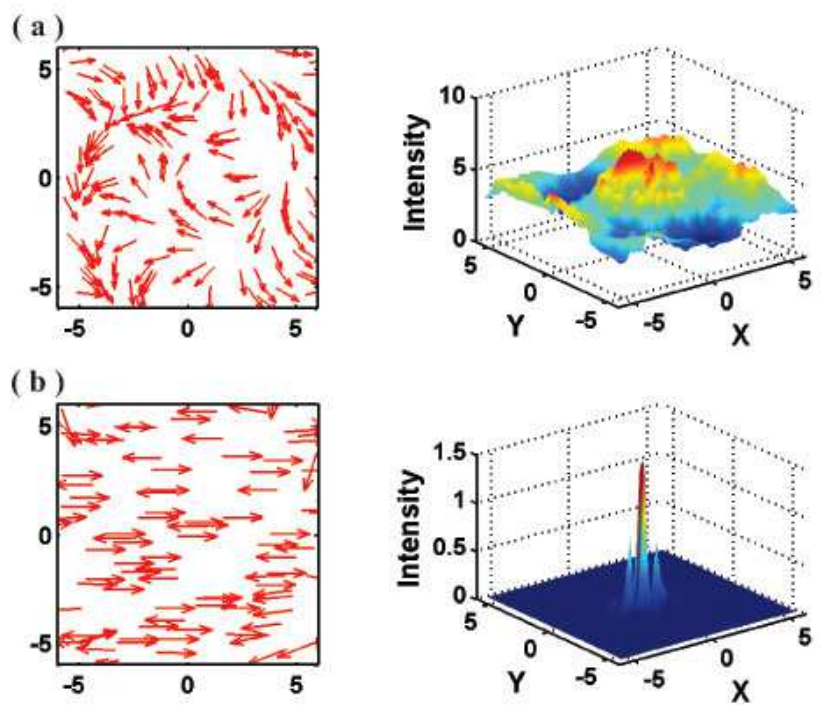

FIG. 3: The phase diagram and spatial distribution of the intensity field $\left|\eta / \eta_{0}\right|^{2}$ at two frequencies for one random configuration: (a1) and (a2) $f=5.0 \mathrm{~Hz}$; (b1) and (b2) $f=$ $11.966 \mathrm{~Hz}$. Left panel: the phase diagram for the phase vectors defined in the text; here the phase of the source is set to zero. Right panel: the intensity spatial distribution in the horizontal plane.

We have also considered the fluctuation in the trans-

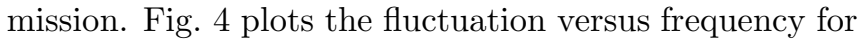
the random case. The sample size $R$ is about $9 \mathrm{~d}$. Here we see that the fluctuation tends to be zero within a regime which is consistent with the strong localization range discussed for Fig. 2 At around the localization transition edges, significant peaks in the transmission fluctuation appear. For extremely low frequencies, the fluctuation tends to disappear. This is because that at when the fre- 
quency approaches zero, the scattering strength will diminish, and thus the wave propagation will be no longer affected by the steps.

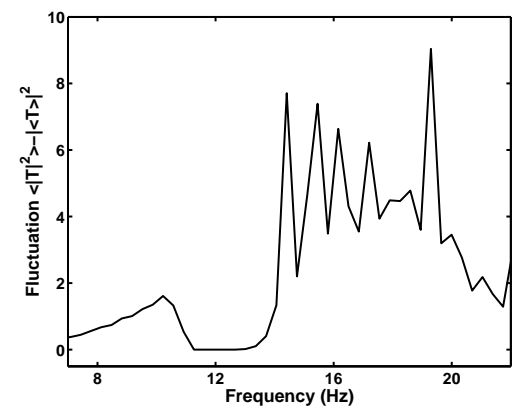

FIG. 4: The fluctuation of transmission as a function of frequency.

To find the localization length, we plot the wave transmission in all directions as a function of the distance from the source. The results are shown in Fig. 5 For comparison, the periodic case is also potted. Here, the simulation data are shown by the black squares, and the results fitted by the least square method is shown by the solid lines; the deviations from the lines reflect the inhomogeneity. It is shown that after the removing the trivial geometrical spreading factor, the data can be fitted well by the exponential function $e^{-r / \xi}$. From the slopes of the solid lines, we obtain the evanescence length in the ordered case and the localization length in the random situation respectively.
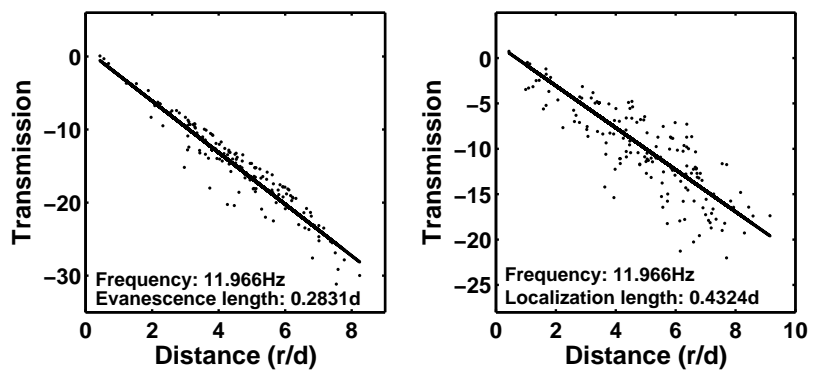

FIG. 5: Wave transmission versus the distance away from the source at $11.966 \mathrm{~Hz}$ : (a) the periodic case; (b) the random case. The geometrical spreading factor has been removed by the normalization. The slopes are fitted from the least square method.

In summary, we have investigated the Anderson localization phenomenon in the framework of gravity waves over rough bottoms. As a comparison, the case of corresponding regularly structured bottoms is also considered. The results indicate that when localization occurs, the wave intensity is confined near the transmitting point, and falls off exponentially. We have also demonstrated that the localized waves stand still in the space, represented by the phase coherence behavior. In addition, the transition from classical diffusion to localization is associated with a significant change in the transmission fluctuation. The observation supports the previous predictions on localized waves. Since water waves are a simple macroscopic system, experiments could be readily performed. Therefore, many significant phenomena, previously expected at microscopic scales such as the discussed Anderson localization, may be demonstrated with water waves.

LSC is supported by the graduate program at Fudan University. The encouragement and support from Prof. $\mathrm{X}$. Sun are greatly appreciated. ZY is grateful to the city of Shanghai for the Bai Yu Lan Fund for visiting scholars.

[1] P. W. Anderson, Phys. Rev. 109, 1492 (1958).

[2] P. Sheng, Introduction to Wave Scattering, Localization, and Mesoscopic Phenomena (Academic Press, New York, 1995).

[3] D. Wiersma, P. Bartolini, A. Lagendijk, R. Righini, Nature 390, 671 (1997).

[4] S. John, Nature, 390, 661 (1997). (1979).

[5] A. Nachbin and G. C. Papanicolaou, J. Fluid Mech. 241, 311 (1992).

[6] T. Chou, Phys. Rev. Lett. 79, 4802 (1997).

[7] Y.-K. Ha, J.-E. Kim, H.-Y. Partk, and I.-W. Lee, Appl. Phys. Lett. 81, 1341 (2002).

[8] P. McIver, Appl. Ocean Res. 24, 121 (2002).

[9] J. H. Pihl, C.-C. Mei, and M. J. Hancock, Phys. Rev. E 66, 016611 (2002); G. L. Gratalop and C.-C. Mei, Phys. Rev. E 68, 026314 (2003).

[10] H. Lamb, Hydrodynamics, (Dover, New York, 1932).

[11] C.-C. Mei, The Applied Dynamics of Ocean Surface Waves, (World Scientific, Singapore, 1989).

[12] M. W. Dingemans, Water Wave Propagation over Uneven Bottoms, (World Scientific, Singapore, 1997).

[13] E. Guazzelli, E. Guyon, and B. Souillard, J. Phys. Lett. 44, L-837 (1983).

[14] P. Devillard, F. Dunlop, and B. Souillard, J. Fluid Mech. 186, 521 (1988).

[15] M. Belzones, E. Guazzelli, and O. Parodi, J. Fluid Mech. 186, 539 (1988).

[16] M. Torres, J. P. Adrados, F. R. Montero de Espinosa, Nature 398, 114 (1999).

[17] M. Torres, et al., Phys. Rev. E 63, 011204 (2000).

[18] Z. Ye, Phys. Rev. E 67, 036623 (2003).

[19] V. Twersky, J. Acoust. Soc. Am. 24, 42 (1951).

[20] S. John, Phys. Rev. Lett. 53, 2169 (1984). 